\title{
Quantifying the rareness of extreme galaxy clusters
}

\author{
Shaun Hotchkiss $*$ \\ Department of Physics, University of Helsinki and Helsinki Institute of Physics, \\ P.O. Box 64, FIN-00014 University of Helsinki, Finland
}

\begin{abstract}
I show that the most common method of quantifying the likelihood that an extreme galaxy cluster could exist is biased and can result in false claims of tension with $\Lambda$ CDM. This common method uses the probability that at least one cluster could exist above the mass and redshift of an observed cluster. I demonstrate the existence of the bias using sample cluster populations, describe its origin and explain how to remove it. I then suggest potentially more suitable and unbiased measures of the rareness of individual clusters. Each different measure will be most sensitive to different possible types of new physics. I show how to generalise these measures to quantify the total 'rareness' of a set of clusters. It is seen that, when mass uncertainties are marginalised over, there is no tension between the standard $\Lambda \mathrm{CDM}$ cosmological model and the existence of any observed set of clusters. As a case study, I apply these rareness measures to sample cluster populations generated using primordial density perturbations with a non-Gaussian spectrum.
\end{abstract}

\section{INTRODUCTION}

Observations of galaxy clusters with sufficiently large masses, at sufficiently large redshifts, can provide strong constraints on possible deviations from the standard $\Lambda$ CDM cosmological model with Gaussian primordial perturbations [1]. Even the observation of a single, sufficiently 'big' and 'early' cluster could rule out the standard model with a high level of confidence [2]. Furthermore, a number of clusters have recently been measured to have masses and redshifts $[3-6]$ that do, even individually, seem to create tension with the combination of $\Lambda \mathrm{CDM}$ and Gaussian initial perturbations [ 7 -9]. When attempts have been made to quantitatively measure the degree to which these clusters are collectively "too big, too early" large discrepancies between $\Lambda \mathrm{CDM}$ and the clusters have been claimed [10, 11]. More of these high mass and high redshift clusters have subsequently been detected by the South Pole Telescope (SPT) [12] and many more will be detected in the near future by SPT, the Planck satellite, the Dark Energy Survey and the XMM Cluster Survey (XCS) [13].

The formation of extreme galaxy clusters depends on both the size of the primordial density perturbations and how long these perturbations have had to grow. In the simplest theoretical models, clusters form when $\delta_{R}$, which is the fractional density contrast, $\delta$, averaged over an approximately spherical region of space with radius $R$, crosses a particular threshold. Due to the assumption of statistical homogenity, at a given time, every point in space has the same probability distibution for $\delta$. Therefore, when $\delta$ is averaged over larger volumes, the fluctuations in $\delta_{R}$ become smaller. This means that regions with large radii are less likely to collapse than regions with small radii. Unfortunately, after collapse it is not possible to directly measure the radius of the region that collapsed to form a cluster. Nevertheless, according to theory, every cluster forms when $\delta_{R}$ crosses one definite threshold. This necessitates that a cluster with a larger mass came from a larger region of space. Therefore, it is expected that fewer clusters will form at larger masses. Fewer clusters are also expected to form at higher redshifts simply because the initial density contrast has had less time to grow. Therefore, the masses and redshifts of observed clusters are good parameters for quantifying the rareness of extreme galaxy clusters. As a result, if it could be shown that the most extreme clusters did form "too big, too early", or even "too little, too late", it would be possible to conclude that either the primordial perturbations do not have a Gaussian spectrum with scale dependence given by a simple power or that the calibration between time and redshift is wrong and therefore clusters did not form as early as is inferred from $\Lambda$ CDM.

It would seem that a very natural method to quantify the rareness of an extreme galaxy cluster is to ask "what is the probability of observing at least one cluster at this mass and redshift or above $(>M>z)$ ?" Once uncertainties in mass measurements, selection functions and cosmological parameters are properly marginalised over this question can then be converted into a measure of the probability that at least one cluster this rare could be observed. It is this resulting measure that has been used to claim that the existence of certain clusters provides tension with $\Lambda$ CDM and a Gaussian, power law, spectrum of density perturbations. This measure is biased. When rareness is measured without bias it is seen that, in $\Lambda \mathrm{CDM}$, there is a greater than $50 \%$ probability that the rarest currently observed cluster could exist somewhere in the fraction of sky observed. Similarly, when the combined rareness of a set of clusters is calculated

\footnotetext{
* shaun.hotchkiss@helsinki.fi
} 
correctly, it is seen that there is a greater than $20 \%$ probability that even the entire set of rarest observed clusters could exist. Nevertheless it is still true that quantifying the rareness of extreme galaxy clusters might provide useful information about possible new physics as will be explored in Section IV.

The core of this paper is structured as follows. In Section III I will discuss the $>M>z$ measure of rareness. I will discuss why to expect that this measure will give biased results. Following this, in Section II A $\mathrm{I}$ will show explicitly that it does lead to biased results when applied to sample cluster populations that were generated assuming a $\Lambda$ CDM universe. In Section III A, I will show how to alter this measure to remove the bias. I will also present other well motivated measures to quantify the rareness of individual clusters. Different measures will be more useful depending on what specific deviations from $\Lambda$ CDM are being tested. In Section IIIB, I will introduce measures that combine the individual rarenesses of a set of clusters to quantify the total rareness of the set as a whole. In both Section III A and Section IIIB. I will apply the introduced measures of rareness to sets of observed galaxy clusters. Finally, in Section[IV] I will apply these rareness measures to sample cluster populations generated from non-Gaussian primordial perturbations.

\section{A. The expected number of clusters in a region of the $(M, z)$ plane}

Before moving on to the core of the paper I will briefly outline the methodology used to calculate the expected number of clusters in a given region of mass and redshift over a given fraction of the sky, $f_{\text {sky. }}$. For each measure of rareness I will be calculating the probabilities of clusters existing assuming a Poissonian distribution; therefore only the expected number is needed. This quantity is given by the following integral

$$
\mathrm{N}=f_{\text {sky }} \int d z \frac{d V}{d z} \int d M \frac{d n(M, z)}{d M}
$$

where the $z$ and $M$ integrals are over the region of the $(M, z)$ plane being considered, $d n / d M$ is the number density of galaxy clusters (the halo mass function) and $d V / d z$ is the volume element,

$$
\frac{d V}{d z}=\frac{4 \pi}{H(z)}\left[\int_{0}^{z} \frac{d z^{\prime}}{H\left(z^{\prime}\right)}\right]^{2} .
$$

It is customary to reparameterise $d n / d M$ as

$$
\frac{d n}{d M}=\frac{\rho_{m}}{M^{2}} \frac{d \ln \sigma(M, z)^{-1}}{d \ln M} f(\sigma, z)
$$

where $\rho_{m}$ is the present density of matter in the universe and $\sigma$ is the variance of the density contrast smoothed over the comoving scale, $R$, that corresponds to the mass, $M$. The function $f$ (often also called the mass function, a convention I will follow in this work) must then either be calculated theoretically or by matching to simulations. The form of $f$ depends on the shape of the primordial spectrum. For most of this work I will be assuming that the primordial spectrum is Gaussian and will use the Tinker et al. mass function [14],

$$
f(\sigma, z)=A\left[\left(\frac{\sigma}{b}\right)^{-a}+1\right] e^{-\frac{c}{\sigma^{2}}}
$$

with $A=0.186(1+z)^{-0.14}, a=1.47(1+z)^{-0.06}, b=2.57(1+z)^{-0.011}$ and $c=1.19$. I will calculate $\sigma(M, 0)$ using the fit to the transfer function presented in [15] with the modified shape parameter introduced in [16]. The redshift dependence of $\sigma$ is then obtained by multiplying $\sigma(M, 0)$ by the linear growth function normalised to be proportional to $1 /(1+z)$ during the era of matter domination. Unless otherwise stated I will also use the WMAP 7 Cosmology maximum likelihood (ML) parameters [17]. Using a different set of cosmological parameters (specifically $\sigma_{8}$ ) would change the exact numbers quoted in parts of this paper but would not alter any of the conclusions drawn from these numbers.

\section{B. Notation used to classify different rareness measures}

In this work I will be introducing a number of different measures to quantify cluster rareness. In each case, the quoted measure is to be interpreted as the probability that at least one cluster (or set of clusters) could exist that is at least as rare as the measured cluster (or set). For reference, I will quickly summarise the notation convention I 


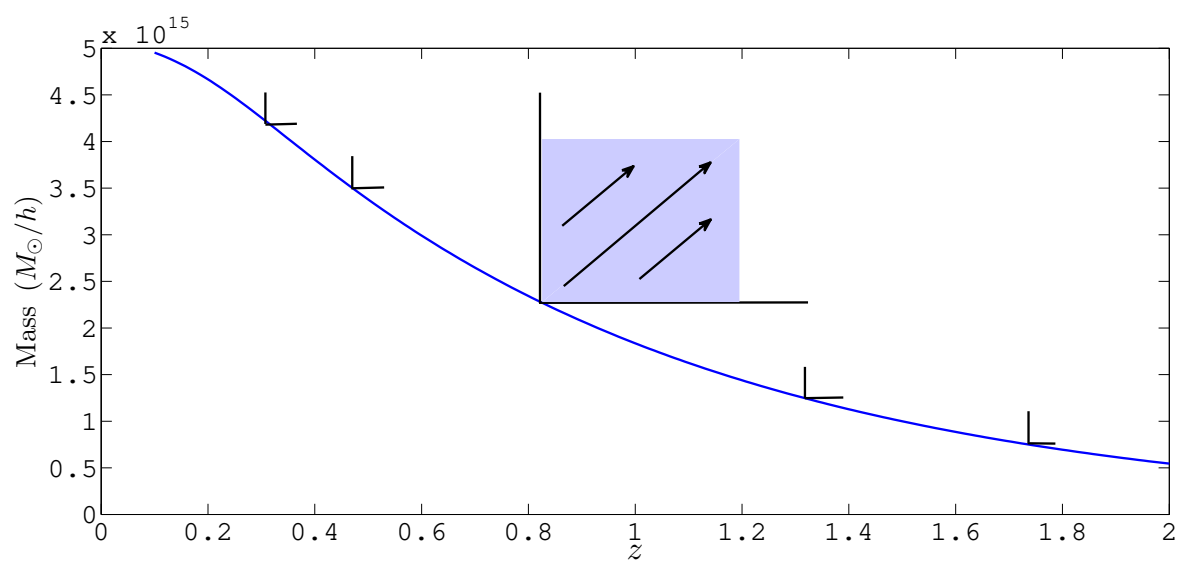

FIG. 1: An outline of the reason for the bias in $\tilde{R}_{>M>z}$. Every point on the curve has the same number of clusters expected at greater masses and redshifts (i.e. the same $\tilde{R}_{>M>z}=\tilde{R}^{*}$ ). The probability that a cluster will exist with $\tilde{R}_{>M>z} \leq \tilde{R}^{*}$ is equal to the probability that a cluster will exist above this entire curve. This probability is necessarily greater than $\tilde{R}^{*}$.

have used. For every measure I use $R$ as the base for an unbiased measure and $\tilde{R}$ for a biased measure. For individual cluster rareness, each measure corresponds to a prescription for defining unique contours in the $(M, z)$ plane. I have chosen to depict the contour used for each measure with subscript text. For example, for the biased version of the $>M>z$ measure of rareness I write $\tilde{R}_{>M>z}$. I also introduce a number of ways to combine the individual rarenesses of a set of clusters. I have chosen to distinguish each method with superscript text. For example, for the method used by Hoyle et al. in Ref.[10] I write $\tilde{R}_{i}^{H}$. To depict the number of clusters used in a combined measure I put this number in subscript immediately before the contour definition. In principle, for a set of clusters, it is possible to combine any contour definition with any method for combining the individual cluster rarenesses.

\section{II. $\tilde{R}_{>M>z}$ AS A MEASURE OF RARENESS}

\section{Why there is a bias}

To quantify the rareness of a cluster using the $>M>z$ measure it is necessary to first calculate the number of clusters expected above the mass and redshift of the observed cluster. This expected number is then converted into the probability that at least one cluster at least as rare as the observed cluster could exist, $\tilde{R}_{>M>z}$, by assuming Poissonian statistics. If this probability is small, the cluster is deemed to be rare and tension can be claimed with $\Lambda \mathrm{CDM}$.

The bias in this measure can be seen in the following way. Using the definition given it is possible to define a value of $\tilde{R}_{>M>z}$ at every point in the $(M, z)$ plane. It is then possible to construct contours of equal $\tilde{R}_{>M>z}$ in the plane. The number of clusters expected above each contour of constant $\tilde{R}_{>M>z}$ is necessarily larger than the expected number of clusters used to calculate $\tilde{R}_{>M>z}$. This is because, for every point in the plane, $\tilde{R}_{>M>z}$ is calculated using only the expected number of clusters at greater masses and greater redshifts. Nevertheless, there will be points at lower masses and greater redshifts (and vice versa) that lie on the same $\tilde{R}_{>M>z}$ contour. These points would not be included in the integration region used to define $\tilde{R}_{>M>z}$ at any other point on the contour, but are defined to be equally rare. Therefore, for a given $\tilde{R}_{>M>z}=\tilde{R}^{*}$, the probability that some cluster exists with $\tilde{R}_{>M>z} \leq \tilde{R}^{*}$ is necessarily greater than $\tilde{R}^{*}$ itself. This is the bias. This argument is illustrated in Figure 1.

\section{The magnitude of the bias}

In Refs. [2, 8] the idea of a contour of constant $\tilde{R}_{>M>z}$ is used explicitly. Ref. [2] provides fitting formulae for the contours as a function of the exclusion limit required to rule out $\Lambda$ CDM. But, for a given exclusion limit of $\alpha$, if any cluster detected above the corresponding contour is to be interpreted as ruling out $\Lambda$ CDM with $100 \alpha \%$ confidence, then the probability of observing such a cluster cannot be greater than $1-\alpha$. In Figure 2 I have tested this. Ref. [2] makes a distinction between $p$, the proportion of WMAP 7 parameter space ruled out, and $s$, the probability to have 


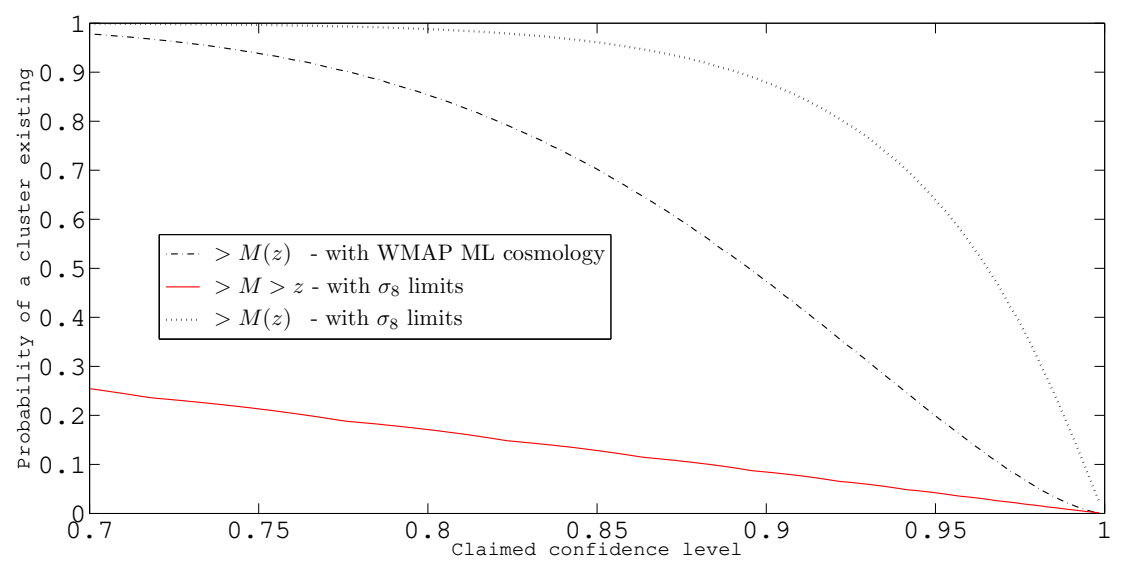

FIG. 2: The solid red line is $\tilde{R}_{>M>z}$, the probability of at least one cluster existing at a greater mass and redshift than each point on a contour, $M(z)$. The dashed and dotted black lines are the probability of at least one cluster existing above the whole $M(z)$ contour. For each data point in the figure, $M(z)$ is defined so that the value of $1-\tilde{R}_{>M>z}$ on $M(z)$ is equal to the value on the x-axis. To produce this figure I have used the fitting formula for $M(z)$ provided in Ref. [2]. See the main text for a discussion of which cosmological parameters were used for each line.

zero clusters $>M>z$ in a random sample of the same sky fraction. I have always taken $s=p$ and have used this as the $\mathrm{x}$-axis of Figure 2. The y-axis gives the probability of at least one cluster existing in a specified region of the $(M, z)$ plane, using a specified set of cosmological parameters. The three different lines correspond to particular regions and particular cosmological parameters. For each line I have taken $f_{\text {sky }}=1$.

The solid red line uses the region of $(M, z)$ defined as $>M>z$ of any arbitrary point on the corresponding contour (by definition all points on the same contour return the same result). Ref.[2] states that, for a contour with a given $p$, in $100 p \%$ of allowed cosmological parameter space the expected number of clusters is less than the expected number that would give the required statistical exclusion limit, $s$. The expected number of clusters depends sensitively and monotonically on $\sigma_{8}$ but very weakly on all other cosmological parameters. Therefore, for the solid red line I calculate the expected number of clusters $>M>z$ using $\sigma_{8}(p)$ defined by

$$
P\left(\sigma_{8}<\sigma_{8}(p)\right)=p .
$$

Following Ref. [2], the distribution for $\sigma_{8}$ was taken from combined CMB, SN, BAO and $H_{0}$ constraints. The red line matches well to expectations indicating that I am correctly interpreting Ref[2]. The dashed and dotted black lines correspond to the probability of observing at least one cluster above the whole $M(z)$ contour. The dashed line comes from using the WMAP maximum likelihood cosmological values and the dotted line comes from using $\sigma_{8}$ defined by eq.(5). From the dashed line it is seen that in a true $\Lambda \mathrm{CDM}$ universe there is a $40 \%$ chance of a cluster existing with a value of $\tilde{R}_{>M>z}$ that would claim to exclude $\Lambda \mathrm{CDM}$ at $90 \%$ confidence. The dotted line makes it possible to estimate the true conservative significance that should be claimed by the observation of a cluster on or above a particular $M(z)$ contour. When the dotted line gives a probability of 0.1 , the $\mathrm{x}$-axis value is $\gtrsim 0.99$. Therefore, if a

cluster were detected that should result in a claim of exclusion at only $\sim 90 \%$ significance, the $\widetilde{\widetilde{R}}_{>M>z}$ statistic would erroneously claim this as $\gtrsim 99 \%$ significance.

\section{A. The bias in practice}

In this section I will examine this bias using sample cluster populations, generated assuming a $\Lambda$ CDM universe. In observations the $>M>z$ contours were not set until the clusters were observed. Therefore, to simulate the observations correctly, it is crucial that the contours are set after the sample is generated, at the masses and redshifts of the sample clusters and not at the masses and redshifts of the clusters observed in our universe.

\section{Individual clusters}

In this subsection I will calculate $\tilde{R}_{>M>z}$ for the most extreme clusters of sample populations. To generate the samples I have first broken the $(M, z)$ plane into bins of equal spacing in $\Delta z$ and $\Delta(\ln M)$. I have then calculated the 

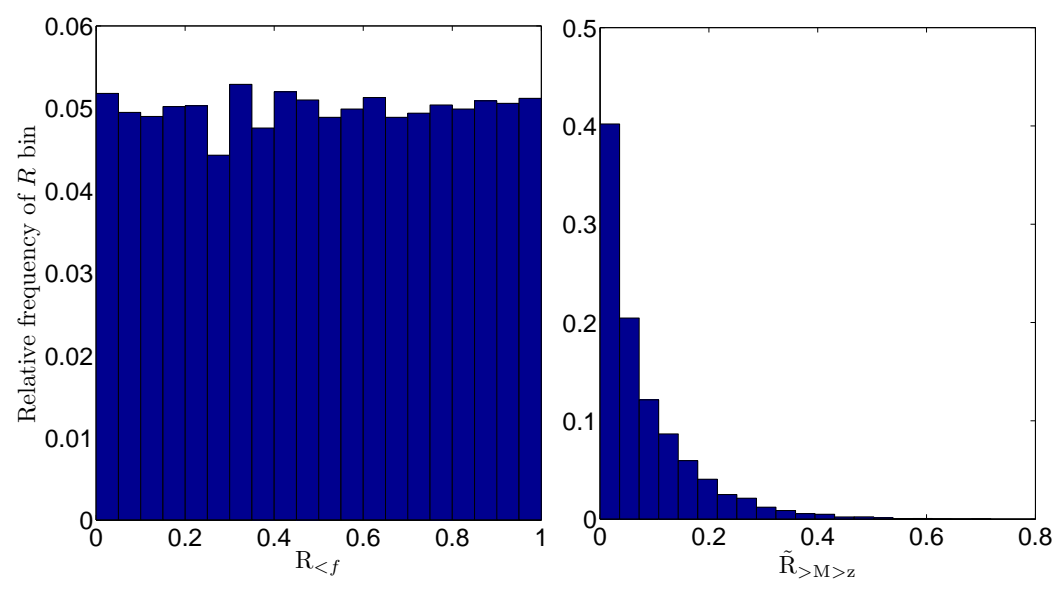

FIG. 3: Histograms showing the distributions generated for an unbiased rareness measure (left panel) and a biased measure (right panel) when they are applied to sample cluster populations. The x-axis on the right panel is the probability that a cluster can exist at greater mass and redshift than the rarest cluster in each sample.

\begin{tabular}{|c|c|c|}
\hline Cluster set & Mean $\tilde{R}_{i>M>z}^{H}$ & Median $\tilde{R}_{i>M>z}^{H}$ \\
\hline \hline H10 & $6.1 \times 10^{-3}$ & $2.6 \times 10^{-6}$ \\
\hline Sample (rarest) & $3.0 \times 10^{-4}$ & $1.5 \times 10^{-6}$ \\
\hline Sample (random) & 0.70 & 0.84 \\
\hline
\end{tabular}

TABLE I: The measure of rareness used in H10 (i.e. Ref. 10]), applied to the table of clusters in H10 and to sample $\Lambda$ CDM cluster populations. This measure was used in [10, 11] to claim significant tension with $\Lambda$ CDM

expected number of clusters to exist in each bin, assuming $\Lambda \mathrm{CDM}$ and using the methodology of Section $[\mathrm{A}$. I then generate a Poisson sample in every bin. The occupied bins determine the masses and redshifts of a sample population of clusters. The bin spacing is ensured to be fine enough such that no bin is ever occupied by more than one cluster.

For every bin in the $(M, z)$ plane I also calculate $\tilde{R}_{>M>z}$. In each sample, I then order the occupied bins with respect to $\tilde{R}_{>M>z}$. The right hand panel of Fig. 3 is a histogram showing the proportion of times that each $\tilde{R}_{>M>z}$ value was the smallest $\tilde{R}_{>M>z}$ value in all of the occupied bin:11. If $\tilde{R}_{>M>z}$ was an unbiased statistic this would be uniform 2; instead, smaller values of $\tilde{R}_{>M>z}$ are disproportionately favoured. The histogram in the left hand panel is an identically produced histogram for one of the unbiased statistics that will be introduced in Section III.

\section{Multiple clusters}

In Refs. 10, 11] the individual $\tilde{R}_{>M>z}$ values for a set of clusters were combined to form an estimate, $\tilde{R}_{i>M>z}^{H}$, of the rareness of the set as a whole. This was done simply by multiplying together the $\tilde{R}_{>M>z}$ values for each of the individual clusters. These references analysed a particular table of clusters put together in Ref. [10] (hereafter referred to as H10). The table consisted, at the time, of all the clusters that had been detected, with spectroscopic follow up, above $z=1$. The masses, redshifts and mass errors for the table of clusters are quoted in Table 1 of both references. Some clusters in this table were detected by X-ray experiments and some from SZ experiments. The convention used in the references was to take $f_{\text {sky }}=283$ sq. degrees for the X-ray detected clusters and $f_{\text {sky }}=178$ for the SZ detected clusters. To compute $\tilde{R}_{i>M>z}^{H}$ for H10 it is necessary to account for mass uncertainties. Both references assumed that the masses of the clusters in the table were log-normally distributed. They then sampled from the mass distribution for each cluster and, for each sample, calculated the corresponding $\tilde{R}_{i>M>z}^{H}$ statistic. The final value of $\tilde{R}_{i>M>z}^{H}$ for the H10 clusters is the mean. The result is very small, $\sim 10^{-3}$, and resulted in claims of large tension.

${ }^{1}$ For this figure I took $f_{\text {sky }}=2500$ sq. degrees and imposed $M>10^{15} M_{\odot}$ (see Section 【II 4 4).

${ }^{2} R$ is supposed to quantify the probability that any cluster could exist that is at least as rare as a given cluster. Put another way, for an unbiased measure, if $R^{*}$ is the rareness of the rarest cluster then $P\left(R^{*} \leq R\right)=R$. This is the definition of a uniform distribution. 


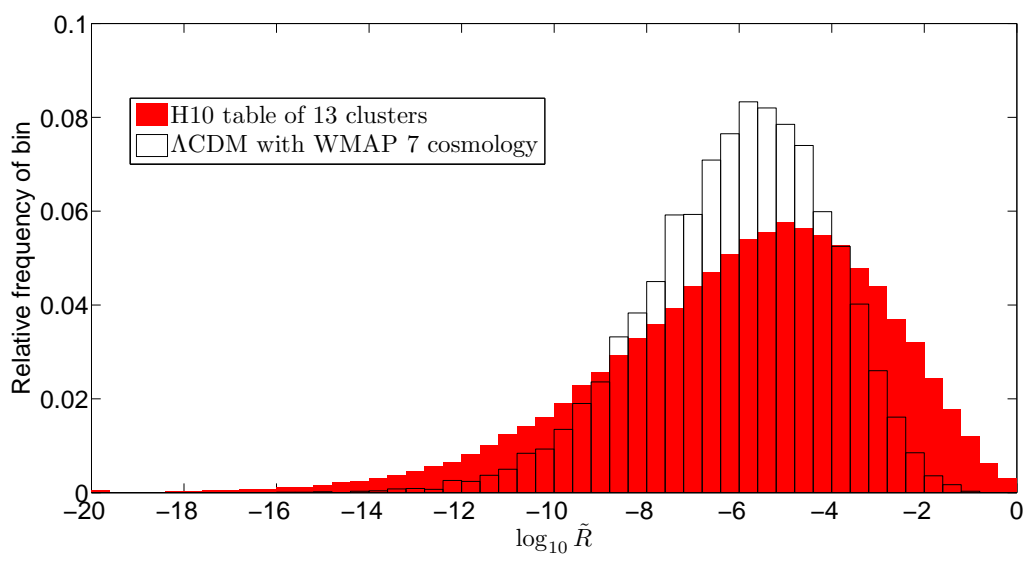

FIG. 4: Histograms of the measure of rareness introduced in H10 applied to the table of clusters in H10 and to sample $\Lambda$ CDM cluster populations. The distribution for H10 comes from sampling the mass uncertainties (see text).

I have repeated this calculation in Table@ with two minor changes. Firstly, I have removed the lightest cluster from H10. This has no effect on the result. Secondly, I have used $f_{\text {sky }}=178+283=461$ sq. degrees for all clusters. This does affect the quoted numbers but not the conclusions that can be drawn from them. This choice was made because it makes it simpler to compare to sample population: 3 , which I have also done. To generate the samples I made a cut of $z>1$ and $M>2 \times 10^{14} M_{\odot}$ to attempt to imitate the nature in which H10 was constructed. Including clusters in the sample below either of these thresholds would increase the effects of the bias by increasing the number of clusters with small $\tilde{R}_{>M>z}$. In Table I the sample (rarest) entry corresponds to the $\tilde{R}_{i>M>z}^{H}$ value calculated using the 13 occupied bins with the smallest $\tilde{R}_{>M>z}$. The sample (random) corresponds to the $\tilde{R}_{i>M>z}^{H}$ value calculated using 13 random occupied bins. It is clear that the mere existence of the H10 clusters does not provide tension with $\Lambda$ CDM. In Fig. 4. I have plotted histograms for H10 and the sample populations. For the samples, the distribution plotted is the proportion of times that a sample had the binned $\tilde{R}_{i>M>z}^{H}$ value using the 13 rarest clusters. For H10, the distribution arises through the log-normal sampling of the mass errors. The distributions are very consistent with each other. In fact, an actual $\Lambda \mathrm{CDM}$ universe would be consistent, to $\sim 95 \%$ confidence, with values of $\tilde{R}_{i>M>z}^{H}$ as small as $10^{-10}$.

Due to the conservative methods used to construct H10 4 it is unlikely that these clusters are the 13 rarest clusters in this $461 \mathrm{sq}$. degree window. If these clusters were picked at random from the sky they would be anomalously rare. However, this also does not reflect the reality. Larger mass clusters are more likely to be seen and the rarest clusters are more interesting and thus more likely to have been spectroscopically followed up. If the full H10 selection function were properly taken into account, the true result for the samples would therefore lie somewhere between the two presented in Table I. This is exactly where the observed value lies.

In Refs. 10, 11] the apparent tension with $\Lambda$ CDM was used to claim detections of minimum values for the nonGaussianity parameters $f_{\mathrm{NL}}$ and $g_{\mathrm{NL}}$. Clearly, without taking selection functions into account, these clusters present no evidence for non-Gaussianity and an unbiased analysis would return results consistent with $f_{\mathrm{NL}}=g_{\mathrm{NL}}=0$.

\section{UNBIASED MEASURES OF RARENESS}

It is easy to test whether any measure of rareness is unbiased by applying it to sample cluster populations. If it returns a uniform distribution, it is unbiased. All of the unbiased measures in this section have been tested in this way. An example of this is shown in the left panel of Figure 3. This was calculated for $R_{<f}$, however any unbiased measure of rareness would give an identical result.

\footnotetext{
${ }^{3}$ It is also arguably the more correct choice. However, treating the SZ and X-ray $f_{\text {sky }}$ values separately is not clearly wrong because not all of the X-ray detected clusters would have been detected by an SZ experiment.

${ }^{4}$ See Ref.[10] for full details, but an example is the already mentioned exclusion of all observed clusters without spectroscopic follow up.
} 


\section{A. Single cluster measures}

$$
\text { 1. } R_{>M>z}
$$

The bias in $\tilde{R}_{>M>z}$ can be removed. This is done simply by finding, for each observed value of $\tilde{R}_{>M>z}$, the true probability of observing this value of $\tilde{R}_{>M>z}$ or less. This amounts to calculating the expected number of clusters above a contour, $M(z)$, of constant $\tilde{R}_{>M>z}$ and then using this expectation value to calculate, $R_{>M>z}$, the probability of at least one cluster at least this rare existing.

In Ref. 9] a cluster was detected that was claimed, using $\tilde{R}_{>M>z}$, to have only a $7 \%$ chance of existing in $\Lambda$ CDM. Using the mass and cosmology quoted in Ref. [9] I find $\tilde{R}_{>M>z}=0.06$ in good agreement with their result. With a mass cut of $M>10^{15} M_{\odot}$ 5 the unbiased value for this cluster is $R_{>M>z}=0.5$. Although this cluster certainly is not a common cluster, its existence does not provide any tension with $\Lambda \mathrm{CDM}$.

$$
\text { 2. } R_{<f}
$$

Despite the fact that $R_{>M>z}$ can be used as an unbiased measure of the rareness of extreme galaxy clusters there are other possible unbiased measures that will be more justified in certain circumstances. The calculation of the expected number of clusters in a region of the $(M, z)$ plane depends on the mass-function, $f$, and the volume element. If it is assumed that the expansion history of the universe is exactly $\Lambda$ CDM but that the primordial perturbations are different then $f$ will change, but the volume element will be unchanged. For this situation, it is useful to define the contours, $M(z)$, of constant rareness as contours of constant $f$. The rareness, $R_{<f}$, is then defined to be the probability that at least one cluster lies above the contour of constant $f$.

This definition would remove the possibly unwanted effect of a cluster being called 'rare' simply because it was found in a region of the $(M, z)$ plane that had a small volume. A region of the $(M, z)$ plane with small $f$ will be seen as rarer by $R_{<f}$ than by $R_{>M>z}$ because regions with larger $f$ but smaller volume will not be included in the expectation value used to calculate $R_{<f}$. If, however, the volume element was different to $\Lambda$ CDM predictions $R_{<f}$ would be less sensitive to this than $R_{>M>z}$.

$$
\text { 3. } R_{>z}
$$

Alternatively, it could be assumed that the primordial perturbations are well described by a Gaussian power law but there are deviations from the $\Lambda \mathrm{CDM}$ volume element or growth function. In this case, the deviations from $\Lambda \mathrm{CDM}$ will depend only on redshift and not on mass at all. For this possibility, it is useful to introduce a measure of rareness that quantifies rareness only by how large $z$ is. Clusters found at large redshift and small mass would be classified as rarer by this measure than they would by other measures.

\section{Measures applied to existing extreme clusters}

I have applied these rareness measures to the clusters in H10 and Ref.[12] (hereafter W11). For the H10 clusters I have used the same redshift cut, mass cut and $f_{\text {sky }}$ as in Section II A 2 If lower masses were allowed, then every cluster would become less rare because more space would be opened up in the $(M, z)$ plane to find clusters. Even with this cut in mass, none of the clusters are exceptionally rare. For the W11 clusters there is no cut in redshift, but a cut of $M>10^{15} M_{\odot}$ and $f_{\text {sky }}=2500$ sq. degrees. This cut is used to approximate the selection function of W11. W11 saw 26 clusters. With this mass cut, the expected number of clusters observed is $>100$. In a more accurate analysis, taking the selection function properly into account, the W11 clusters would be rarer than what I quote here. However, the same calculation with a higher mass cut, designed to produce only 26 expected clusters, gives qualitatively the same results; that is, none of the clusters are exceptionally rare. I have presented results for $M>10^{15} M_{\odot}$ because this is the lowest mass of any of the clusters observed in W11.

Table Џ lists $R_{<f}$ and $R_{>M>z}$ for the most extreme H10 and W11 clusters. Uncertainties in the masses of the clusters have been accounted for with the method described in Section $\amalg_{1}{ }^{6}$ In this table I have also presented the

\footnotetext{
5 This cluster is also in W11 (see Section [II A 4). The mass quoted in Ref. [9] is larger than in W11 for reasons explained in Ref. [9].

${ }^{6}$ For the W11 clusters I added the experimental and sytematic errors in quadrature.
} 


\begin{tabular}{|c||c|c|c|c|}
\hline Cluster & $R_{<f}$ & $R_{>M>z}$ & $\langle f$ Mass at $z=0$ & $>M>z$ Mass at $z=0$ \\
\hline \hline $\mathrm{J} 2235.3+2557(\mathrm{H} 10)$ & 0.58 & 0.49 & $7.7 \times 10^{15} M_{\odot}$ & $3.3 \times 10^{15} M_{\odot}$ \\
\hline J0546-5345 (H10) & 0.76 & 0.61 & $6.2 \times 10^{15} M_{\odot}$ & $2.8 \times 10^{15} M_{\odot}$ \\
\hline J0910+5422(H10) & 0.86 & 0.79 & $4.5 \times 10^{15} M_{\odot}$ & $1.8 \times 10^{15} M_{\odot}$ \\
\hline $\mathrm{J} 2215.9-1738(\mathrm{H} 10)$ & 0.85 & 0.81 & $5.2 \times 10^{15} M_{\odot}$ & $1.8 \times 10^{15} M_{\odot}$ \\
\hline \hline $\mathrm{J} 0102-4915(\mathrm{~W} 11)$ & 0.63 & 0.61 & $7.1 \times 10^{15} M_{\odot}$ & $3.8 \times 10^{15} M_{\odot}$ \\
\hline $\mathrm{J} 0615-5746(\mathrm{~W} 11)$ & 0.63 & 0.70 & $7.1 \times 10^{15} M_{\odot}$ & $3.5 \times 10^{15} M_{\odot}$ \\
\hline $\mathrm{J} 0658-5556(\mathrm{~W} 11)$ & 0.84 & 0.63 & $5.2 \times 10^{15} M_{\odot}$ & $3.6 \times 10^{15} M_{\odot}$ \\
\hline $\mathrm{J} 2106-5844(\mathrm{~W} 11)$ & 0.73 & 0.86 & $6.7 \times 10^{15} M_{\odot}$ & $3.0 \times 10^{15} M_{\odot}$ \\
\hline $\mathrm{J} 2248-4431(\mathrm{~W} 11)$ & 0.84 & 0.66 & $5.3 \times 10^{15} M_{\odot}$ & $3.5 \times 10^{15} M_{\odot}$ \\
\hline $\mathrm{J} 2344-4243(\mathrm{~W} 11)$ & 0.92 & 0.88 & $5.0 \times 10^{15} M_{\odot}$ & $2.7 \times 10^{15} M_{\odot}$ \\
\hline
\end{tabular}

TABLE II: Two unbiased rareness measures applied to the most extreme clusters in H10 and W11. As well as, each cluster's 'equivalent mass at redshift zero' according to both rareness measures. This is the mass of a cluster at redshift zero that would be judged to be equally as rare as the actual observed cluster. The value of $R$ should be interpreted as the probability that at least one cluster could exist that is at least as rare as the observed cluster.

"equivalent mass at redshift zero" of each cluster. As seen in the previous section, an unbiased measure of rareness is constructed from a set of contours in the $(M, z)$ plane defined to be equally rare. The "equivalent mass at redshift zero" is then the point at which the $M(z)$ contour for a given cluster and measure crosses zero. That is, another cluster observed at redshift zero, with mass $M(0)$ would be judged to be equally rare. For these values I have chosen not to marginalise over the mass uncertainties, using only the central value of mass quoted in H10 and W11. The actual equivalent mass at redshift zero depends on the rareness measure. The more intuitively sensible values are those for the $R_{>M>z}$ measure. The $R_{<f}$ measure, while useful when looking for new physics, loses something here because it neglects the volume element, which is small at $z=0$. While some of these clusters would be very massive at $z=0$, they would not be beyond precedent 7 .

Although no single cluster in Table $\amalg$ is extremely rare, it might be thought that the total probability of having this many clusters with rareness $\lesssim 0.7$ would be very small. However, caution must be taken when estimating the rareness of a set of clusters by looking at the rareness of its constituents, especially when mass uncertainties are large. For any cluster, large mass uncertainties will push $R$ towards 0.5 because the uncertainties will make the cluster more consistent with being both very rare $(R \simeq 0)$ and very generic $(R \simeq 1)$. When considering a set of clusters, the mass uncertainties need to be taken into account all at once. If the uncertainties are large then the same effect will drive $R$ towards 0.5 for the set as well. Thus, two individual $R$ 's close to 0.5 should not necessarily be interpreted as meaning the two clusters are rare as an ensemble. It is possible that they are both just not well measured. Nevertheless, two clusters with very small mass uncertainties and rareness measures of $\sim 0.5$ would be seen to be much rarer as an ensemble. The following section will answer this question decisively for H10 and W11. Neither set is particularly rare.

\section{B. Multiple cluster measures}

Studying the rareness of individual galaxy clusters is useful and may provide information about new physics. However it may be the case that any deviations from $\Lambda \mathrm{CDM}$ are subtle. Thus, no individual galaxy cluster might be rare enough on its own to rule out $\Lambda$ CDM to a high degree of significance. Perhaps, if there are a number of clusters, which individually provide only small tension with $\Lambda \mathrm{CDM}$, they might collectively provide much more tension. To quantify this it is necessary to have a measure of the rareness of a set of clusters.

Quantifying the rareness of a set of clusters risks potential biases similar to the individual rareness biases. Consider the situation where only two clusters are being examined, clusters $A$ and $B$. An intuitive rareness measure, $\tilde{R}^{T}$, is constructed by asking "what is the probability of at least one cluster existing that is at least as rare as $A$ and at least one cluster existing that is at least as rare as $B$ ?". This measure would be biased. If $A$ was made less rare but $B$ was made more rare to compensate then $\tilde{R}^{T}$ can remain the same. Therefore, the true probability of a pair of clusters

\footnotetext{
7 Care should be taken when comparing the masses for H10 and for W11. Because of the different cuts in mass and redshift, equal values of $f$ or $\tilde{R}_{>M>z}$, for clusters from different sets, would not equate to equal values of $R_{<f}$ and $R_{>M>z}$.
} 
existing that are as rare as $A$ and $B$ is necessarily greater than $\tilde{R}^{T}$. With sets of clusters care must also be taken because the rareness of one cluster is not independent of the rareness of other clusters in the set. Nevertheless, these obstacles can be overcome. I present below three possible methods.

$$
\text { 1. } \tilde{R}^{H}
$$

$\tilde{R}^{H}$ was introduced in Section $\llbracket A 2$ where it was biased because I used it with $\tilde{R}_{>M>z}$. It would also be biased if instead I used $R_{>M>z}$. This second bias results from the method of multiplying the individual cluster rareness together. Distributions that are uniform between zero and one, will not give a uniform distribution when multiplied together. The benefit of this measure is that it is easy to calculate. Any result can be compared to sample cluster populations to check expectations and thus remove the bias.

$$
\text { 2. } R^{M}
$$

Another simple, multiple cluster measure of rareness is suggested in Ref.[2]. This measure asks what is the probability that there are at least $i$ clusters at least as rare as the $i$ th rarest cluster in the set. This gives

$$
R_{i}^{M}=1-e^{-\lambda_{i}} \sum_{n}^{i} \frac{\lambda_{i}^{n}}{n !}
$$

where $\lambda_{i}$ is the expected number of clusters above the contour of constant rareness for the $i^{\text {th }}$ rarest cluster. $R^{M}$ is unbiased because it depends on only the one parameter, $\lambda_{i}$.

$$
\text { 3. } R^{C}
$$

It could occur that all the $R_{i}^{M}$ are small but not small enough to claim a significant deviation from $\Lambda$ CDM. Alternatively, one of the $R_{i}^{M}$ could be very small, but all the rest $\gtrsim 0.1$. What is needed is a way to quantify the combined significance of all of the $R_{i}^{M}$. $\tilde{R}^{H}$ will do this to a certain degree, but crudely. Most of the effect on $\tilde{R}^{H}$ comes from the one or two most extreme clusters whereas $R_{i}^{M}$ could be significant at large $i$.

The easiest suggestion is to multiply each of the $R_{i}^{M}$ together. This is fine and would work (it is biased, but the bias is easily accounted for). However, the individual $R_{i}^{M}$ measures are not independent of each other. If $R_{1}^{M}$ is known to be very small then this enhances the probability that $R_{2}^{M}$ will also be small. If the two individual measures are naively multiplied together then two sets of clusters that are not equally rare will be classified so. This weakens the sensitivity of the measure.

The solution to this is to use a measure defined as the probability of the intersection of the rareness measures.

$$
\tilde{R}_{i}^{C}=P\left(R_{1}^{M}<R_{1(\mathrm{obs})}^{M} \cap R_{2}^{M}<R_{2(\mathrm{obs})}^{M} \cap \cdots \cap R_{i}^{M}<R_{i(\mathrm{obs})}^{M}\right),
$$

where $R_{i \text { (obs) }}^{M}$ is the observed value of $R_{i}^{M}$. By definition, $R_{1}^{C}=R_{1}^{M}$. The $i=2$ calculation proceeds as follows. First, define $N_{i}$ to be the number of clusters, in a sample universe, above the contour of constant rareness associated to the observed $i^{\text {th }}$ rarest cluster. Define $N_{i j}$ to be the number of clusters between the $i^{\text {th }}$ and $j^{\text {th }}$ contours. Then

$$
\begin{aligned}
\tilde{R}_{2}^{C} & =P\left(N_{2} \geq 2 \cap N_{1} \geq 1\right) \\
& =P\left(N_{12} \geq 0\right) \times P\left(N_{1} \geq 2\right)+P\left(N_{12} \geq 1\right) \times P\left(N_{1}=1\right) \\
& =P\left(N_{1} \geq 1\right)+P\left(N_{12}=0\right) \times P\left(N_{1}=1\right) \\
& =R_{1}^{C}-\lambda_{1} e^{-\lambda_{2}},
\end{aligned}
$$

which is true because $N_{12}=N_{2}-N_{1}$ and $P(N \geq n)=1-P(N<n)$. Similar calculations for $\tilde{R}_{3}^{C}$ and $\tilde{R}_{4}^{C}$ return

$$
\begin{aligned}
& R_{1}^{C}=1-e^{-\lambda_{1}}, \\
& \tilde{R}_{2}^{C}=R_{1}^{C}-\lambda_{1} e^{-\lambda_{2}}, \\
& \tilde{R}_{3}^{C}=\tilde{R}_{2}^{C}-\lambda_{1} e^{-\lambda_{3}}\left(\lambda_{2}-\frac{\lambda_{1}}{2}\right), \\
& \tilde{R}_{4}^{C}=\tilde{R}_{3}^{C}-e^{-\lambda_{4}}\left(\frac{\lambda_{1}^{3}}{6}+\lambda_{1} \lambda_{2} \lambda_{3}-\frac{\lambda_{1} \lambda_{2}^{2}}{2}-\frac{\lambda_{3} \lambda_{1}^{2}}{2}\right) .
\end{aligned}
$$




\begin{tabular}{|c||c|c|c|c|c|c|c|c|c|c|}
\hline$i$ & 1 & 2 & 3 & 4 & 5 & 6 & 7 & 8 & 9 & 10 \\
\hline \hline$R_{i<f}^{M}$ & 0.32 & 0.30 & 0.33 & 0.43 & 0.57 & 0.72 & 0.83 & 0.90 & 0.94 & 0.97 \\
\hline$R_{i>M>z}^{M}$ & 0.22 & 0.21 & 0.23 & 0.28 & 0.36 & 0.44 & 0.53 & 0.62 & 0.71 & 0.78 \\
\hline
\end{tabular}

TABLE III: The rareness of the $i^{\text {th }}$ rarest cluster in W11, using two different methods to define the contours of constant rareness in the $(M, z)$ plane. The value of $R$ should be interpreted as the probability that the $i^{\text {th }}$ rarest existing cluster is at least as rare as the $i^{\text {th }}$ rarest W11 cluster.

These rareness measures are of course biased because they depend on more than one parameter. The bias can be removed by comparing observations to sample cluster populations. It can also be removed by a much more efficient calculation. It is actually only the $\lambda_{i}$ that we need to know the distributions for and not the full $(M, z)$ distribution of all clusters in the sky. The distribution for $\lambda_{1}$ is easily constructed because the distribution of $R_{1}^{M}=1-\exp \left(-\lambda_{1}\right)$ is uniform. It might be hoped that all of the $\lambda_{i}$ could be constructed from the corresponding, uniform $R_{i}^{M}$ distribution. If all that was needed was the distribution of one $\lambda_{i}$ on its own this would be fine. However, what is actually needed is the conditional distribution for $\lambda_{i}$ given the values of all the other $\lambda_{j}$ with $j \neq i$. These distributions can be sampled with the following method. First, allocate a value to $\lambda_{1}$ using a uniform distribution for $R_{1}^{M}$. This assigns the contour of the rarest cluster. Next it is necessary to assign the contour of the second rarest cluster. The probability of observing at least one cluster between the rarest contour and the second rarest contour is

$$
R_{12}^{M}=1-e^{-\lambda_{12}}
$$

where $\lambda_{12}=\lambda_{2}-\lambda_{1}$. A value can be allocated to $\lambda_{12}$ using a uniform distribution for $R_{12}^{M}$. This determines $\lambda_{2}$ and assigns the contour of the second rarest cluster. The same process will work for every $\lambda_{i}$ by first determining $\lambda_{i-1}$ and then allocating a value to $\lambda_{i(i-1)}=\lambda_{i}-\lambda_{i-1}$ using the uniform distribution, $R_{i(i-1)}^{M}=1-\exp \left(-\lambda_{i(i-1)}\right)$. This method is much more efficient than simulating an entire cluster population (with $>10^{4}$ bins) and then finding the $i$ rarest clusters. Using the distributions for $\lambda_{i}$, the bias in $\tilde{R}^{C}$ is removed by calculating the probability that $\tilde{R}^{C}$ will be less than the value observed.

$$
R^{C}=P\left(\tilde{R}^{C}<\tilde{R}_{(\text {obs })}^{C}\right)
$$

For $i \leq 4$, I have tested this method for calculating $R_{i}^{C}$ by applying it to sample cluster populations. It is unbiased and produces a uniform distribution.

\section{Measures applied to observations}

Although none of the individual clusters in H10 and W11 are significantly rare I speculated in Section 1 AI 4 that perhaps the sets as a whole might be. It is now possible to check. In Table III. I have listed $R_{i>M>z}^{M}$ and $R_{i<f}^{M}$ for the W11 clusters. I have used the method for accounting for mass uncertainties described in Section II A 2 . To clear up a possible ambiguity, I sample once from each mass distribution in the entire W11 set, I then calculate the individual rareness of every cluster, pick the $i$ rarest and then calculate the combined rareness. I do not pre-assign, before making each sample, which are the rarest clusters. I use the same mass cut, redshift cut and $f_{\text {sky }}$ as I did for the W11 entries of Table II.

For the first few values of $i$, both contour definitions indicate that the rarest W11 clusters are slightly rarer than the $\Lambda$ CDM average, but not with any statistical significance - there is at least a $20 \%$ chance of a set of clusters as rare as W11 occurring in $\Lambda$ CDM. As $i$ increases, $R$ approaches 1 for both contour definitions. This indicates that, for the mass cut I used and for $i \gtrsim 6$, the $i^{\text {th }}$ rarest clusters in W11 are not as rare as they should be. This could be an indication of new physics; however it is much, much more likely to be the result of neglecting selection function effects. I have already noted that with the mass cut I use, more than 100 clusters are expected to exist. There are only 26 clusters in W11. SPT do not expect to see every cluster that exists above the mass cut I have imposed. Therefore, it is probable that the expected but missing rare clusters do exist, but are near this mass threshold, rather than absent due to reasons of fundamental physics. The reason that selection function effects are more pronounced for larger values of $i$ is that larger mass clusters are both more likely to be seen and more rare. Therefore, it is more likely that the rarest cluster in any survey has been seen than, for example, the 5th rarest cluster.

In Table IV I have listed $R_{i<f}^{C}$, with $i \leq 4$, for both W11 and H10. The method to account for mass uncertainties is exactly equivalent to that for Table III and the mass cut, redshift cut and $f_{\text {sky }}$ is the same for each set as it was for Table II Table IV points to the same conclusion as Table III. The total significance of the four rarest clusters shows 


\begin{tabular}{|c||c|c|c|c|}
\hline$i$ & 1 & 2 & 3 & 4 \\
\hline \hline W11 & 0.32 & 0.30 & 0.30 & 0.31 \\
\hline H10 & 0.29 & 0.31 & 0.33 & 0.36 \\
\hline
\end{tabular}

TABLE IV: The combined rareness, $R_{i<f}^{C}$, of the $i$ rarest clusters in H10 and W11. The value of $R$ should be interpreted as the probability that a set of $i$ clusters could exist that is at least as rare as the $i$ rarest clusters in H10 or W11.

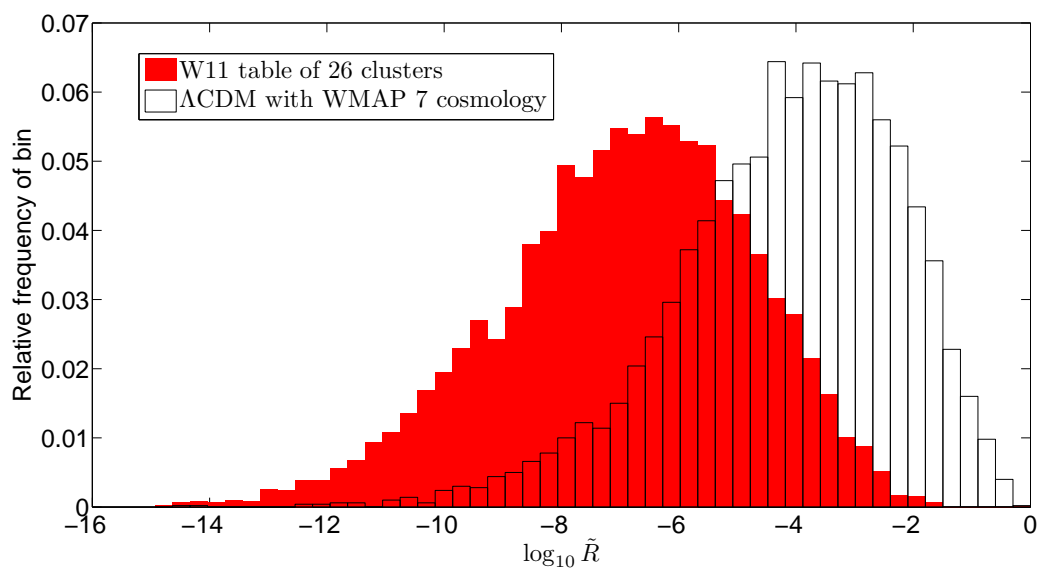

FIG. 5: Histogram of the rareness measure introduced in H10 applied to both the W11 clusters and sample $\Lambda$ CDM populations. This is equivalent to Figure 4, but for W11 instead of H10.

that sets of clusters as rare as H10 and W11 would occur in $\gtrsim 30 \%$ of $\Lambda \mathrm{CDM}$ universes. This could mean that we live in a $\Lambda \mathrm{CDM}$ universe, or simply that the mass errors in both sets are too large to distinguish any new physics.

Finally, in Figure 5. I have plotted histograms of $\tilde{R}_{26>M>z}^{H}$ for W11 and for sample cluster populations with the same mass cut and $f_{\text {sky }}$ as I have used for W11. The median value for $\mathrm{W} 11$ is $\tilde{R}=1.7 \times 10^{-7}$. This value or less occurs for $\sim 0.3$ of the simulated populations. This is consistent with what was seen using the other rareness measures in Tables [II and IV. The equivalent figure for H10 is Figure 4. Figure 4 seems to indicate the H10 clusters are not rare enough for $\Lambda \mathrm{CDM}$, whereas Table IV indicated that they were slightly too rare. Note however that the $i$ values in Table IV peak at $i=4$, whereas Figure 4 is for all 13 clusters in H10. As already discussed, it is very unlikely that the $\mathrm{H} 10$ ensemble really has seen the 13th rarest cluster in its observation window, whereas it is quite possible that it has seen the rarest four. An equivalent figure to Figure 4 including only the four rarest clusters in H10 and the sample cluster populations returns a result consistent with Table IV. This effect probably was not seen in the comparable W11 analysis because of its more complete survey. 8 .

To go further in the analysis would require modelling of the selection function for each set of clusters. Given that both sets are slightly rarer than a $\Lambda \mathrm{CDM}$ average and the fact that accounting for the selection function could only make the observed clusters appear rarer, this might be an interesting exercise. Although, accounting for cosmological uncertainties would weaken the strength of any obtainable result. To make an estimate of the effects of selection functions I have attempted re-doing the analyses presented here with a larger mass cut for W11. If I set the mass cut to give an expected number of clusters of 26, the measured rareness of W11 did become more significant, but $R^{C}$ did not fall below 0.05 . However, if selection functions are to be properly taken into account, any analysis might as well go beyond just measuring the rareness of the most extreme clusters and measure instead the total goodness of fit of all the clusters observed. In the regions of sky considered by W11 and H10, many more clusters have been observed than are actually analysed. This is because both references made cuts $(z>1$ for H10 and significance of detection ( mass) for W11) that isolate the most extreme clusters. The purpose of measuring rareness is to find a way to quantify the likelihood of these most extreme observed clusters existing. It is less useful as a total goodness of fit measure for 100's of clusters.

\footnotetext{
${ }^{8}$ W11 has 26 of the $\sim 100$ clusters expected after my choice of mass cut - H10 has only 13 of the $\sim 300$ expected. Note that even in Table IV an increase in $R_{i}^{C}$ is noticeable for $i=4$.
} 


\begin{tabular}{|c||c|c|c||c|c|c|}
\hline \multicolumn{1}{|c||}{} & \multicolumn{3}{c||}{$R_{i<f}^{C}$} & \multicolumn{3}{c|}{$R_{i<f}^{M}$} \\
\hline$i$ & $f_{\mathrm{NL}}=50$ & $f_{\mathrm{NL}}=100$ & $f_{\mathrm{NL}}=500$ & $f_{\mathrm{NL}}=50$ & $f_{\mathrm{NL}}=100$ & $f_{\mathrm{NL}}=500$ \\
\hline \hline 1 & 0.39 & 0.30 & 0.022 & 0.40 & 0.30 & 0.023 \\
\hline 2 & 0.37 & 0.26 & 0.0050 & 0.38 & 0.24 & 0.0024 \\
\hline 3 & 0.36 & 0.24 & 0.0012 & 0.34 & 0.20 & $3.0 \times 10^{-4}$ \\
\hline 4 & 0.34 & 0.21 & $3.1 \times 10^{-4}$ & 0.32 & 0.17 & $4.5 \times 10^{-5}$ \\
\hline
\end{tabular}

TABLE V: The probability that the rarest clusters in a non-Gaussian universe could exist in a Gaussian universe. $R_{i}^{M}$ quantifies the rareness of the $i^{\text {th }}$ rarest cluster. $R_{i}^{C}$ quantifies the combined rareness of all of the $i$ rarest clusters.

\section{RARENESS APPLIED TO PRIMORDIAL NON-GAUSSIANITY}

As a case study of the use of rareness measures I will apply some of the measures of the previous section to sample cluster populations arising from non-Gaussian primordial density perturbations. No attempts have been made to consider the effects of selection functions, cosmological uncertainties or mass errors. The benefit of measuring the rareness of sample populations is that this is not necessary. Therefore, although the results are instructive of what would happen in a statistics limited experiment, they are not representative of any near-future experiments. A more accurate analysis of the constraints that observations of a small number of extreme galaxy clusters could provide on non-Gaussianity must be left for future work.

The method used to generate cluster populations from non-Gaussian primordial density perturbations is identical to that used for Gaussian primordial density perturbations except for the use of the mass function. I have chosen to replace the Tinker et al. mass function used earlier with the prescription for a non-Gaussian mass function outlined in [18]. This prescription provides a formula for the ratio between the full non-Gaussian mass function and the Gaussian mass function. The prescription then dictates that the formula for the ratio is to be multiplied by a mass function trusted in the Gaussian limit. There are many prescriptions in the literature that each give slightly different formulae for the ratio. To within the accuracy and range of masses, redshifts and quantity of non-Gaussianity currently tested by N-body simulations, they agree with each other. I have chosen this particular prescription because it is expected to be more stable (and therefore more accurate) at the larger masses, redshifts and quantities of non-Gaussianity that have not yet been tested by simulation. This seems appropriate given that I am examining the most extreme objects in sample cluster populations. As in Ref.[18] and earlier sections of this work, for the Gaussian limit I will use the Tinker et al. mass function. This prescription parameterises the non-Gaussianity using the 'local' template which has the one parameter, $f_{\mathrm{NL}}[19]$.

In Table $\mathrm{V}$. I have calculated $R_{i<f}^{M}$ and $R_{i<f}^{C}$. To do this I have used $f_{\text {sky }}=1$ and made a mass cut of $M>10^{14} M_{\odot}$ and a redshift cut of $z>0.1$. I have generated sample cluster populations using the quoted value of $f_{\mathrm{NL}}$ and then asked how rare each population would be in $\Lambda$ CDM. I have not included $f_{\mathrm{NL}}=0$ in the table because by construction it returns 0.5 for every $R$. Clearly, for large enough $f_{\mathrm{NL}}$, observation of even the rarest four galaxy clusters could rule out $\Lambda \mathrm{CDM}$. Disappointingly, for smaller $f_{\mathrm{NL}}$ this is not the case. Nonetheless, the two measures do behave as expected. $R_{i}^{C}$ should quantify the total combined significance of the the first $i R_{i}^{M}$ measures. This seems to be what is happening in the table. $R_{i<f}^{M}$ drops as $i$ increases, indicating that each individual statistic is further from $\Lambda$ CDM. $R_{i<f}^{C}$ drops appropriately, although not as rapidly because it 'remembers' the weaker significance of $R_{j}^{M}$ for all $j<i$.

The final result of this work is Figure 6 which has histograms of $\tilde{R}_{i>M>z}^{H}$ for $f_{\mathrm{NL}}=100$ and $\Lambda \mathrm{CDM}$. This result matches what is seen in Table $\mathrm{V}$ for $f_{\mathrm{NL}}=100$. This shows consistency amongst the various rareness measures using different contours of constant rareness and different means to combine individual rareness.

\section{SUMMARY AND DISCUSSION}

In this work I have examined how to accurately quantify the rareness of extreme galaxy clusters. I have shown that the most typical method, $\tilde{R}_{>M>z}$, is biased and makes clusters appear less likely to exist than they actually are. The easiest way to understand this is the fact that the probability of a cluster existing with a given value of $\tilde{R}_{>M>z}$ is greater than $\tilde{R}_{>M>z}$ itself. Therefore, the event of observing a cluster with a small value of $\tilde{R}_{>M>z}$ is not necessarily expected to be an uncommon event. For example, in an actual $\Lambda$ CDM universe, there is a $\sim 40 \%$ probability of a cluster existing that would be claimed by $\tilde{R}_{>M>z}$ to rule out $\Lambda$ CDM at $90 \%$ significance. I have also shown this bias explicitly by calculating $\tilde{R}_{>M>z}$ for sample cluster populations. 


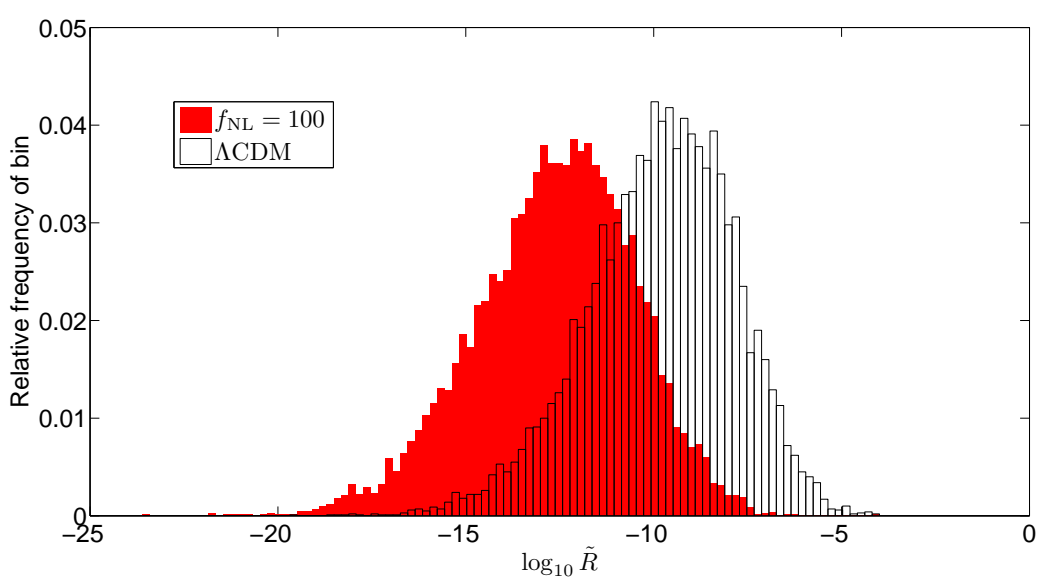

FIG. 6: Histograms of the rareness measure introduced in H10 applied to a population of Gaussian $\Lambda$ CDM universes and to a population of universes with $f_{\mathrm{NL}}=100$.

I have shown how to remove the bias in $\tilde{R}_{>M>z}$. I have also presented other unbiased measures of the rareness of individual clusters, $R_{<f}$ and $R_{>z}$. This set is not exhaustive of all possible measures. In fact, any prescription that defines a unique set of $M(z)$ contours in the $(M, z)$ plane will define a corresponding measure of rareness. For example, asking what the probability is that a given cluster is the most massive cluster in a survey window would be another possible rareness measure (see [20]). I have argued that $R_{<f}$ will be the measure that is most susceptible to deviations from $\Lambda \mathrm{CDM}$ arising through changes in the primordial sepctrum of density perturbations and that $R_{>z}$ will be most susceptible to deviations in the expansion history and growth function of the primordial perturbations.

I have presented methods to combine the individual rarenesses of a set of clusters into a combined measure of the 'rareness' of the set. $\tilde{R}_{i}^{H}$, first used in Hoyle et al.[10], simply multiplies together the individual rarenesses of $i$ clusters. This method of combining rarenesses introduces its own bias; however the bias can be straightforwardly accounted for by comparing to sample populations. The most signficant flaw in $\tilde{R}^{H}$ is that it is more sensitive to the rareness of the most extreme cluster, than it is to any other cluster in a set. $R_{i}^{M}$, first used in Mortonson et al. [2], instead quantifies the probability that the $i^{\text {th }}$ rarest cluster in the universe could be as rare as the $i^{\text {th }}$ rarest in a given set. $R_{i}^{M}$ can then be calculated at each value of $i$ to look for possible deviations from $\Lambda$ CDM. However, it could occur that $R_{i}^{M}$ is small for all $i$, but never small enough to claim significant tension, or $R_{i}^{M}$ could be significantly small for some values of $i$, but not for others. $R_{i}^{C}$, first presented here, quantifies the combined rareness of all of the $i$ rarest clusters. I have presented formulae for a biased value of $R_{i}^{C}$ (i.e. $\tilde{R}_{i}^{C}$ ) for $i \leq 4$ and a computationally efficient method to account for this bias (without the need to generate sample cluster populations). $R_{i}^{C}$ includes all the independent information regarding rareness that is contained in the masses and redshifts of the $i$ rarest clusters.

I have applied these individual and combined rareness measures to the sets of clusters found in Ref. [10] (H10) and Ref.[12] (W11). The result is that each of the clusters has a greater than $50 \%$ probability of existing in a $\Lambda$ CDM universe. There is also a greater than $20 \%$ probability that a set of clusters as rare as either H10 or W11 could exist in $\Lambda$ CDM. These constraints are conservative and could be improved by accounting for the selection function of each sample and reducing the mass uncertainties. For the most extreme clusters in each sample I have also listed the "equivalent mass at redshift zero", which is the mass of a cluster at $z=0$ that would be judged to be equally rare. While some of the clusters would be quite large at $z=0$ they would not be without precedent.

As a working example I applied the rareness measures to sample cluster populations generated using a non-Gaussian primordial spectrum. For very large non-Gaussianity, even the four rarest clusters could in principle rule out the standard cosmological model with high significance. For more realistic levels of non-Gaussianity, a small number of the most extreme clusters will not be able to provide significant tension. Note however that my analysis has used the non-Gaussianity parameter, $f_{\mathrm{NL}}$. It is expected that the abundance of extreme clusters (and voids) will be relatively more susceptible to higher order non-Gaussianity parameters (e.g. $g_{\mathrm{NL}}, \tau_{\mathrm{NL}}$ ) than $f_{\mathrm{NL}}$ (see for example Refs. [11, 21]).

In Ref. [2] convenient fitting formulae were provided for $M(z)$ contours as a function of $f_{\text {sky }}$ and the exclusion limit with which one desires to rule out $\Lambda \mathrm{CDM}$ (using $\tilde{R}_{>M>z}$ ). Unfortunately, generalising these contours to unbiased measures of rareness is not straightforward. The major problem is that the region that it is necessary to integrate over in the $(M, z)$ plane to unbiasedly quantify rareness is experiment dependent. If an experiment cannot see clusters below a certain mass threshold, a cluster observed by this experiment should not be claimed to be 'likely' because equivalently rare clusters might exist below this threshold. A very conservative set of fitting formulae could be developed by assuming that an experiment can see everything in the $(M, z)$ plane; however this would probably be 
so conservative that it would effectively be impotent. Therefore, any accurate fitting formulae would also need to depend on observational cuts made in the $(M, z)$ plane.

When calculating the rareness of observed galaxy clusters my aim was to show that previously claimed sources of tension were a result of the use of the biased measure $\tilde{R}_{>M>z}$. Sometimes, because $\tilde{R}_{>M>z}$ was so small, the previous works in the literature made very conservative assumptions elsewhere in their analysis. I have also made these conservative assumptions. It is possible that a less conservative analysis of the rareness of observed galaxy clusters could still show significant tension with $\Lambda$ CDM. This is especially true of the set of clusters in H10 (in constructing their table, H10 excluded clusters without a spectroscopic redshift measurement, deliberately chose mass estimates that had large errors, were conservative in their choice of $f_{\text {sky }}$ and neglected any selection function effects). However, to avoid accidentally making false claims of tension, any future analysis will need to either account for the bias in $\tilde{R}_{>M>z}$, use one of the naturally unbiased measures presented here, or, choose a new, well motivated, prescription for a set of equal rareness contours in the $(M, z)$ plane.

Note:

During the preparation of this manuscript, Ref. [22] was posted to the arXiv. In Ref. 22], the gravitational lensing masses of 27 high-redshift clusters are presented. Ref.[22] claims to find significant tension with $\Lambda$ CDM; however they do this using the biased measures $\tilde{R}_{>M>z}$ and $\tilde{R}_{4>M>z}^{H}$. With a preliminary analysis, using $f_{\text {sky }}=100$ sq. degrees, I do not find that any individual cluster in Ref. 22] provides tension with $\Lambda$ CDM. Also, for the set of clusters in Ref. 22], I find $R_{4<f}^{C}=0.19$ (and similar values for $i<4$ ). Tantalisingly, however, I find the following sequence of values for $R_{i<f}^{M}: 0.30,0.24,0.13,0.072,0.055,0.061,0.092,0.37,0.50$ and 0.58 . Unfortunately I have not yet developed the machinery to evaluate $R_{i}^{C}$ for $i>4$ to test the actual significance of this curious trend.

\section{ACKNOWLEDGEMENTS}

I thank Kari Enqvist, Chris Gordon, Ben Hoyle, Aseem Paranjape and Olli Taanila for helpful comments relating to an earlier draft of this work. I also thank Ben Hoyle for useful discussions during the early stages of this work and Syksy Rasanen for some late advice. The seed of the idea for this work came from a post relating to Ref. [9] by Fergus Simpson at the Cosmo Coffee website. I acknowledge the support of Academy of Finland grant 131454.

[1] S. W. Allen, A. E. Evrard and A. B. Mantz, arXiv:1103.4829 [astro-ph.CO].

[2] M. J. Mortonson, W. Hu and D. Huterer, Phys. Rev. D 83 (2011) 023015 [arXiv:1011.0004 [astro-ph.CO]].

[3] C. R. Mullis, P. Rosati, G. Lamer, H. Bohringer, A. Schwope, P. Schuecker and R. Fassbender, Astrophys. J. 623 (2005) L85 arXiv:astro-ph/0503004.

[4] T. Broadhurst, K. Umetsu, E. Medezinski, M. Oguri and Y. Rephaeli, Astrophys. J. 685, L9 (2008) arXiv:0805.2617 [astro-ph]].

[5] A. Mantz, S. W. Allen, H. Ebeling, D. Rapetti and A. Drlica-Wagner, Mon. Not. Roy. Astron. Soc. 406 (2010) 1773 arXiv:0909.3099 [astro-ph.CO]].

[6] J. Richard, J. P. Kneib, M. Limousin, A. Edge and E. Jullo, arXiv:0910.5553 [astro-ph.CO].

[7] M. J. Jee et al., Astrophys. J. 704, 672 (2009) arXiv:0908.3897 [astro-ph.CO]].

[8] D. E. Holz and S. Perlmutter, arXiv:1004.5349 [astro-ph.CO].

[9] R. J. Foley et al., Astrophys. J. 731 (2011) 86 [arXiv:1101.1286 [astro-ph.CO]].

[10] B. Hoyle, R. Jimenez and L. Verde, arXiv:1009.3884 [astro-ph.CO].

[11] K. Enqvist, S. Hotchkiss and O. Taanila, JCAP 1104, 017 (2011) arXiv:1012.2732 [astro-ph.CO]].

[12] R. Williamson et al., arXiv:1101.1290 [astro-ph.CO].

[13] E. J. Lloyd-Davies et al., arXiv:1010.0677] [astro-ph.CO].

[14] J. L. Tinker et al., Astrophys. J. 688 (2008) 709 arXiv:0803.2706 [astro-ph]].

[15] J. M. Bardeen, J. R. Bond, N. Kaiser and A. S. Szalay, Astrophys. J. 304 (1986) 15.

[16] N. Sugiyama, Astrophys. J. Suppl. 100 (1995) 281 arXiv:astro-ph/9412025.

[17] E. Komatsu et al. [WMAP Collaboration], Astrophys. J. Suppl. 192 (2011) 18 arXiv:1001.4538 [astro-ph.CO]].

[18] A. Paranjape, C. Gordon and S. Hotchkiss, arXiv:1104.1145 [astro-ph.CO].

[19] E. Komatsu and D. N. Spergel, Phys. Rev. D 63 (2001) 063002 arXiv:astro-ph/0005036.

[20] L. Cayon, C. Gordon and J. Silk, arXiv:1006.1950 [astro-ph.CO].

[21] S. Chongchitnan and J. Silk, Astrophys. J. 724 (2010) 285 arXiv:1007.1230 [astro-ph.CO]].

[22] M. J. Jee et al., arXiv:1105.3186 [astro-ph.CO]. 